\title{
Erratum to: Evaluation of the best linear unbiased prediction method for breeding values of fruit-quality traits in citrus
}

\author{
A. Imai ${ }^{1,2,3}$ - T. Kuniga ${ }^{1,4} \cdot$ T. Yoshioka $^{1,5} \cdot$ K. Nonaka $^{1} \cdot$ N. Mitani ${ }^{1,6}$ • \\ H. Fukamachi ${ }^{1}$ - N. Hiehata ${ }^{1,7}$ • M. Yamamoto ${ }^{1,8}$ - T. Hayashi ${ }^{3,9}$
}

Published online: 30 December 2016

(C) Springer-Verlag Berlin Heidelberg 2016

Erratum to: Tree Genetics \& Genomes (2016) 12: 119.

DOI: $10.1007 / \mathbf{s} 11295-016-1078-8$

Unfortunately, all of the tables (Tables 1, 2, 3, 4, 5, and 6) in the original version of this article were misprinted in the process of proof reading. The correct tables are reprinted below with their legends.

\section{Data Archiving Statement}

All relevant data of the original article are presented in this errata and in the Supplementary Materials of the original article.

The online version of the original article can be found under doi: 10.1007 /s11295-016-1078-8.

T. Hayashi

hayatk@affrc.go.jp

1 Citrus Research Division, National Agriculture and Food Research Organization (NARO) Institute of Fruit Tree Science (NIFTS),

Minamishimabara, Nagasaki 859-2501, Japan

2 Breeding and Pest Management Division, National Agriculture and Food Research Organization (NARO) Institute of Fruit Tree Science (NIFTS), Tsukuba, Ibaraki 305-8605, Japan

3 Graduate School of Life and Environmental Sciences, University of Tsukuba, Tsukuba, Ibaraki 305-8666, Japan

4 Hillside Horticulture Research Division, National Agriculture and Food Research Organization (NARO) Western Region Agricultural Research Center (WARC), Zentsuji, Kagawa 765-0053, Japan
5 Citrus Research Division, National Agriculture and Food Research Organization (NARO) Institute of Fruit Tree Science (NIFTS), Shimizu, Shizuoka 424-0292, Japan

6 Division of Fruit Production and Postharvest Science, National Agriculture and Food Research Organization (NARO) Institute of Fruit Tree Science (NIFTS), Tsukuba, Ibaraki 305-8605, Japan

7 Kenou Development Bureau, Nagasaki Prefectural Government, Isahaya, Nagasaki 854-0071, Japan

8 Faculty of Agriculture, Kagoshima University, Korimoto, Kagoshima 890-0065, Japan

9 Division of Agricultural Systems and Information Technology, National Agriculture and Food Research Organization (NARO) National Agricultural Research Center (NARC), Tsukuba, Ibaraki 305-8666, Japan 
Table 1 List of parental cultivars/genotypes and their usage frequencies in the Kuchinotsu Citrus Breeding Program at the National Institute of Fruit Tree Science (NIFTS-KCBP) from 2009 to 2012

\begin{tabular}{|c|c|c|c|}
\hline $\mathrm{Name}^{\mathrm{a}}$ & Species or parentage ${ }^{\mathrm{b}}$ & Accession $^{\mathrm{c}}$ & No. of crosses ${ }^{\mathrm{d}}$ \\
\hline satsuma mandarin & Citrus unshiu Marcow. & 'Okitsu wase'(170630) & 9 \\
\hline clementine & Citrus clementina hort. ex Tanaka & Stock strain (113161) & 1 \\
\hline sweet orange & Citrus sinensis (L.) Osbeck & 'Trovita'(172154) & 2 \\
\hline iyo & Citrus iyo hort. ex Tanaka & 'Miyauchi'(117374) & 0 \\
\hline hyuga-natsu & Citrus tamurana hort. ex Tanaka & Stock strain (117317) & 2 \\
\hline grapefruit & Citrus paradisi Macfad. & 'Duncan'(168864) & 2 \\
\hline dancy tangerine & Citrus tangerina hort. ex Tanaka & Stock strain (117396) & 0 \\
\hline king mandarin & Citrus nobilis Lour. & Stock strain (117386) & 1 \\
\hline mediterranean mandarin & Citrus deliciosa Ten. & Stock strain (117393) & 0 \\
\hline ponkan & Citrus reticulata Blanco & 'Yoshida'(113178) & 5 \\
\hline kishu mandarin & Citrus kinokuni hort. ex Tanaka & 'Mukaku-kishu'(171490) & 0 \\
\hline hassaku & Citrus hassaku hort. ex Tanaka & Stock strain (115524) & 0 \\
\hline Tanikawa-buntan & unknown origin & Stock strain (117433) & 0 \\
\hline San-jacinto & unknown origin & Stock strain $(113350)$ & 1 \\
\hline Soren-tangelo & unknown origin & Stock strain (113347) & 1 \\
\hline tankan & Citrus tankan Hayata & 'Tarumizu 1 Gou'(113508) & 7 \\
\hline Murcott & unknown origin & Stock strain (113374) & 0 \\
\hline Nankou & satsuma mandarin $\times$ clementine & Stock strain $(117160)$ & 8 \\
\hline HF15 & satsuma mandarin $\times$ sweet orange & Stock strain (n.a.) & 0 \\
\hline HF17 & satsuma mandarin $\times$ sweet orange & Stock strain (n.a.) & 0 \\
\hline $\mathrm{HF} 20$ & satsuma mandarin $\times$ sweet orange & Stock strain (n.a.) & 0 \\
\hline $\mathrm{HF} 21$ & satsuma mandarin $\times$ sweet orange & Stock strain (n.a.) & 0 \\
\hline HF9 & satsuma mandarin $\times$ sweet orange & Stock strain (n.a.) & 1 \\
\hline Aki Tangor & satsuma mandarin $\times$ sweet orange & Stock strain (n.a.) & 7 \\
\hline Kiyomi & satsuma mandarin $\times$ sweet orange & Stock strain (115521) & 3 \\
\hline Minneola & grapefruit $\times$ dancy tangerine & Stock strain (113377) & 0 \\
\hline Orlando & grapefruit $\times$ dancy tangerine & Stock strain (113327) & 0 \\
\hline Encore & king mandarin $\times$ mediterranean mandarin & Stock strain $(117421)$ & 0 \\
\hline Wilking & king mandarin $\times$ mediterranean mandarin & Stock strain (117425) & 1 \\
\hline Ariake & sweet orange $\times$ clementine & Stock strain $(117158)$ & 0 \\
\hline Sweet Spring & satsuma mandarin $\times$ hassaku & Stock strain (168866) & 1 \\
\hline No. 1087 & iyo $\times$ sweet orange & Stock strain (n.a.) & 0 \\
\hline Awa Orange & hyuga-natsu $\times$ sweet orange & Stock strain (115757) & 0 \\
\hline JHG & satsuma mandarin $\times$ hyuga-natsu & Stock strain (n.a.) & 3 \\
\hline Kara & satsuma mandarin $\times$ king mandarin & Stock strain (113158) & 4 \\
\hline Hayaka & satsuma mandarin $\times$ ponkan & Stock strain (117154) & 0 \\
\hline Kankitsu Chukanbohon Nou 6 Gou & king mandarin $\times$ Mukaku-kishu & Stock strain $(223661)$ & 4 \\
\hline Southern Yellow & Tanikawa-buntan $\times$ Mukaku-kishu & Stock strain $(117470)$ & 3 \\
\hline Nishinokaori & Kiyomi $\times$ sweet orange & Stock strain (118841) & 2 \\
\hline Osceola & clementine $\times$ Orlando & Stock strain (113329) & 0 \\
\hline Lee & clementine $\times$ Orlando & Stock strain (113385) & 0 \\
\hline Robinson & clementine $\times$ Orlando & Stock strain (113386) & 0 \\
\hline KyOw14 & Kiyomi $\times$ satsuma mandarin & Stock strain (n.a.) & 4 \\
\hline KyOw21 & Kiyomi $\times$ satsuma mandarin & Stock strain (n.a.) & 0 \\
\hline EnOw20 & Encore $\times$ satsuma mandarin & Stock strain (n.a.) & 0 \\
\hline EnOw21 & Encore $\times$ satsuma mandarin & Stock strain (n.a.) & 1 \\
\hline
\end{tabular}


Table 1 (continued)

\begin{tabular}{|c|c|c|c|}
\hline $\mathrm{Name}^{\mathrm{a}}$ & Species or parentage ${ }^{\mathrm{b}}$ & Accession $^{\mathrm{c}}$ & No. of crosses ${ }^{\mathrm{d}}$ \\
\hline EnOw7 & Encore $\times$ satsuma mandarin & Stock strain (n.a.) & 0 \\
\hline EnOw8 & Encore $\times$ satsuma mandarin & Stock strain (n.a.) & 0 \\
\hline Shiranuhi & Kiyomi $\times$ ponkan & Stock strain (117159) & 14 \\
\hline Setomi & Kiyomi $\times$ ponkan & Stock strain (223670) & 5 \\
\hline Harumi & Kiyomi $\times$ ponkan & Stock strain (117468) & 6 \\
\hline Youkou & Kiyomi $\times$ ponkan & Stock strain (n.a.) & 0 \\
\hline Page & clementine $\times$ Minneola & Stock strain (113370) & 0 \\
\hline No. 2681 & Kiyomi $\times$ iyo & Stock strain (n.a.) & 0 \\
\hline Tsunonozomi & Kiyomi $\times$ Encore & Stock strain (n.a.) & 6 \\
\hline KyEn4 & Kiyomi $\times$ Encore & Stock strain (n.a.) & 0 \\
\hline KyEn5 & Kiyomi $\times$ Encore & Stock strain (n.a.) & 1 \\
\hline Amaka & Kiyomi $\times$ Encore & Stock strain (118843) & 0 \\
\hline Tamami & Kiyomi $\times$ Wilking & Stock strain (223657) & 4 \\
\hline F118 & Kiyomi $\times$ Wilking & Stock strain (n.a.) & 0 \\
\hline Okitsu 45 Gou & Kiyomi $\times$ Wilking & Stock strain (n.a.) & 0 \\
\hline Benibae & HF9 $\times$ Encore & Stock strain (n.a.) & 10 \\
\hline HF9· En29 & HF9 $\times$ Encore & Stock strain (n.a.) & 0 \\
\hline A7 & Sweet Spring $\times$ sweet orange & Stock strain (n.a.) & 0 \\
\hline Okitsu 46 Gou & Sweet Spring $\times$ sweet orange & Stock strain (n.a.) & 2 \\
\hline Hareyaka & Encore $\times$ satsuma mandarin & Stock strain $(117510)$ & 0 \\
\hline M5 & Kiyomi $\times$ dancy tangerine & Stock strain (n.a.) & 0 \\
\hline $2700 \cdot$ Oly 25 & Nishinokaori $\times$ iyo & Stock strain (n.a.) & 0 \\
\hline E647 & Kiyomi $\times$ Osceola & Stock strain (n.a.) & 6 \\
\hline KyOw21 · D4 & KyOw $21 \times$ dancy tangerine & Stock strain (n.a.) & 0 \\
\hline KyOw21 · D49 & KyOw $21 \times$ dancy tangerine & Stock strain (n.a.) & 0 \\
\hline Kuchinotsu 28 Gou & KyOw $21 \times$ dancy tangerine & Stock strain (n.a.) & 0 \\
\hline Kuchinotsu 27 Gou & EnOw21 × Youkou & Stock strain (n.a.) & 6 \\
\hline Seinannohikari & EnOw $21 \times$ Youkou & Stock strain (n.a.) & 8 \\
\hline Amakusa & KyOw14 × Page & Stock strain (117161) & 1 \\
\hline Kuchinotsu 33 Gou & KyOw14 × Encore & Stock strain (n.a.) & 2 \\
\hline Tsunokagayaki & KyOw $14 \times$ Encore & Stock strain (n.a.) & 12 \\
\hline Kuchinotsu 18 Gou & KyOw21 × Encore & Stock strain (n.a.) & 0 \\
\hline Kuchinotsu 35 Gou & KyOw21 × Encore & Stock strain (n.a.) & 3 \\
\hline Kuchinotsu 38 Gou & KyOw21 × Robinson & Stock strain (n.a.) & 1 \\
\hline No. 1408 & EnOw $21 \times$ No. 2681 & Stock strain (n.a.) & 1 \\
\hline Kankitsu Chukanbohon Nou 5 Gou & Lee $\times$ Mukaku-kishu & Stock strain $(223660)$ & 1 \\
\hline 990343 & Tsunonozomi $\times$ Benibae & Stock strain (n.a.) & 2 \\
\hline Kanpei & Nishinokaori $\times$ Shiranuhi & Stock strain (n.a.) & 5 \\
\hline Okitsu 57 Gou & Okitsu 46 Gou $\times$ Harumi & Stock strain (n.a.) & 15 \\
\hline Asumi & Okitsu 46 Gou $\times$ Harumi & Stock strain (n.a.) & 3 \\
\hline Okitsu 59 Gou & $\mathrm{A} 7 \times \mathrm{F} 118$ & Stock strain (n.a.) & 1 \\
\hline Setoka & Tsunonozomi $\times$ Murcott & Stock strain (118842) & 5 \\
\hline KyEn5 $\cdot$ En6 & KyEn $5 \times$ Encore & Stock strain (n.a.) & 0 \\
\hline Reikou & KyEn5 × Murcott & Stock strain (n.a.) & 0 \\
\hline Kuchinotsu 36 Gou & KyEn5 × Murcott & Stock strain (n.a.) & 3 \\
\hline $\mathrm{KyOw} 21 \cdot \mathrm{CC} 33$ & KyOw $21 \times$ clementine & Stock strain (n.a.) & 0 \\
\hline
\end{tabular}


Table 1 (continued)

\begin{tabular}{|c|c|c|c|}
\hline $\mathrm{Name}^{\mathrm{a}}$ & Species or parentage ${ }^{\mathrm{b}}$ & Accession $^{\mathrm{c}}$ & No. of crosses ${ }^{\mathrm{d}}$ \\
\hline KyOw21 $\cdot$ Ariake22 & KyOw $21 \times$ Ariake & Stock strain (n.a.) & 0 \\
\hline Kuchinotsu 40 Gou & KyOw $21 \times$ No. 1087 & Stock strain (n.a.) & 1 \\
\hline Haruhi & Okitsu 46 Gou $\times$ Awa Orange & Stock strain (n.a.) & 1 \\
\hline LeeAo25 & Lee $\times$ satsuma mandarinLee $\times$ satsuma mandarin & Stock strain (n.a.) & 0 \\
\hline LeeAo35 & Lee $\times$ satsuma mandarinLee $\times$ satsuma mandarin & Stock strain (n.a.) & 0 \\
\hline LeeAo9 & Lee $\times$ satsuma mandarinLee $\times$ satsuma mandarin & Stock strain (n.a.) & 0 \\
\hline No. 1010 & Nankou $\times 2700 \cdot$ Oly 25 & Stock strain (n.a.) & 0 \\
\hline No. 1011 & Nankou $\times 2700 \cdot$ OIy 25 & Stock strain (n.a.) & 7 \\
\hline Harehime & E647 $\times$ satsuma mandarin & Stock strain (n.a.) & 9 \\
\hline Kuchinotsu 51 Gou & KyOw21 $\cdot$ D4 × Kuchinotsu 27 Gou & Stock strain (n.a.) & 1 \\
\hline No. 1051 & HF9 $\times 2700 \cdot$ Oly 25 & Stock strain (n.a.) & 0 \\
\hline Ehime Kashi No. 28 & Nankou $\times$ Amakusa & Stock strain (n.a.) & 5 \\
\hline Kuchinotsu 52 Gou & Tsunokagayaki $\times$ Ariake & Stock strain (n.a.) & 0 \\
\hline 960203 & Kuchinotsu 18 Gou $\times$ Youkou & Stock strain (n.a.) & 3 \\
\hline Kuchinotsu 49 Gou & Kuchinotsu 38 Gou $\times$ No. 1408 & Stock strain (n.a.) & 6 \\
\hline Mihaya & Tsunonozomi $\times$ No. 1408 & Stock strain (n.a.) & 8 \\
\hline 980389 & HF15 × No. 1408 & Stock strain (n.a.) & 7 \\
\hline Okitsu 56 Gou & Okitsu 45 Gou $\times$ Kankitsu Chukanbohon Nou 5 Gou & Stock strain (n.a.) & 6 \\
\hline 031060 & Harehime $\times$ clementine & Stock strain (n.a.) & 2 \\
\hline
\end{tabular}

${ }^{a}$ Names in bold represent founding genotypes of NIFTS-KCBP, consisting of 13 known species and four genotypes of unknown origin

${ }^{\mathrm{b}}$ Regarding 13 species in founding genotypes, we selected a representative cultivar within each species for phenotypic evaluation

c Accessions used for phenotypic evaluation. Numbers in parentheses represent the accession ID (JP number) in the NIAS germplasm collection. Accessions with "n.a." have not been registered in the collection

d Number of crosses that the parent was involved in within 126 pair-cross families used in this study. " 0 " indicates their descendant genotypes were used as cross-parents

Table 2 The nine fruit-quality traits evaluated in this study

\begin{tabular}{lll}
\hline Trait & Data type & Measurement unit \\
\hline Fruit weight & Continuous & Mean weight of mature fruits (g) \\
Fruit skin color & Ordinal & $1:$ yellow, 2:orange, 3:red \\
Fruit surface texture & Ordinal & $1:$ rough, 2:medium, 3:smooth \\
Peelability & Ordinal & $1:$ difficult, 2:medium, 3:easy \\
Flesh color & Ordinal & $1:$ :ellow, 2:light-orange, 3:orange \\
Pulp firmness & Ordinal & $1:$ firm, 2:medium, 3:soft \\
Segment firmness & Ordinal & $1:$ firm, 2:medium, 3:soft \\
Sugar content & Continuous & Mean Brix of juice (Brix\%) \\
Acid content & Continuous & Mean citric acid concentration of juice (\%) \\
\hline
\end{tabular}


Table 3 Summary statistics based on yearly means of individual genotypes of the nine fruit-quality traits evaluated in this study

\begin{tabular}{|c|c|c|c|c|c|c|c|c|c|c|}
\hline \multirow[t]{2}{*}{ Year } & \multirow[t]{2}{*}{ Descriptive statistics ${ }^{\mathrm{a}}$} & \multicolumn{9}{|l|}{ Traits } \\
\hline & & FW (g) & FSC & FST & $\mathrm{PE}$ & $\mathrm{FC}$ & $\mathrm{PF}$ & $\mathrm{SF}$ & $\mathrm{SC}(\mathrm{Brix} \%)$ & $\mathrm{AC}(\%)$ \\
\hline \multirow[t]{5}{*}{2009} & Mean & 139.3 & 2.12 & 2.47 & 2.60 & 2.94 & 2.69 & 2.64 & 11.9 & 1.20 \\
\hline & S.D. & 61.7 & 0.46 & 0.61 & 0.61 & 0.26 & 0.62 & 0.60 & 1.6 & 0.55 \\
\hline & Min & 29.7 & 1 & 1 & 1 & 1 & 1 & 1 & 8.4 & 0.46 \\
\hline & Max & 626.0 & 3 & 3 & 3 & 3 & 3 & 3 & 16.6 & 3.37 \\
\hline & Records & 422 & 418 & 421 & 421 & 421 & 346 & 346 & 421 & 421 \\
\hline \multirow[t]{5}{*}{2010} & Mean & 152.9 & 2.13 & 2.53 & 2.57 & 2.95 & 2.67 & 2.62 & 12.0 & 1.29 \\
\hline & S.D. & 66.3 & 0.39 & 0.66 & 0.65 & 0.25 & 0.57 & 0.61 & 1.5 & 0.55 \\
\hline & Min & 30.6 & 1 & 1 & 1 & 1 & 1 & 1 & 8.2 & 0.54 \\
\hline & Max & 621.5 & 3 & 3 & 3 & 3 & 3 & 3 & 18.3 & 4.16 \\
\hline & Records & 619 & 618 & 618 & 618 & 618 & 618 & 617 & 617 & 614 \\
\hline \multirow[t]{5}{*}{2011} & Mean & 190.2 & 2.13 & 2.46 & 2.48 & 2.92 & 2.59 & 2.54 & 10.9 & 1.22 \\
\hline & S.D. & 81.4 & 0.43 & 0.68 & 0.72 & 0.28 & 0.62 & 0.63 & 1.4 & 0.54 \\
\hline & Min & 38.2 & 1 & 1 & 1 & 1 & 1 & 1 & 7.4 & 0.41 \\
\hline & Max & 949.5 & 3 & 3 & 3 & 3 & 3 & 3 & 16.2 & 3.83 \\
\hline & Records & 640 & 639 & 639 & 640 & 640 & 637 & 639 & 640 & 640 \\
\hline \multirow[t]{5}{*}{2012} & Mean & 154.1 & 2.01 & 2.34 & 2.38 & 2.84 & 2.58 & 2.52 & 11.6 & 1.47 \\
\hline & S.D. & 59.7 & 0.51 & 0.71 & 0.78 & 0.41 & 0.66 & 0.62 & 1.3 & 0.66 \\
\hline & Min & 32.8 & 1 & 1 & 1 & 1 & 1 & 1 & 7.7 & 0.58 \\
\hline & Max & 541.0 & 3 & 3 & 3 & 3 & 3 & 3 & 16.2 & 4.48 \\
\hline & Records & 1782 & 1780 & 1781 & 1781 & 1781 & 1781 & 1780 & 1777 & 1778 \\
\hline
\end{tabular}

$F W$ fruit weight, $F S C$ fruit skin color, $F S T$ fruit surface texture, $P E$ peelability, $F C$ flesh color, $P F$ pulp firmness, $S F$ segment firmness, $S C$ sugar content, $A C$ acid content

${ }^{a}$ The means represent raw means unadjusted for the combination of remaining effects and are different in genotype for each year.

The other summary statistics also differ with genotype for each year.

Table 4 Best linear unbiased estimator (BLUE) values of intercepts and year effects for nine fruit-quality traits

\begin{tabular}{|c|c|c|c|c|c|}
\hline \multirow[t]{2}{*}{ Traits } & \multicolumn{5}{|c|}{ Best linear unbiased estimator (BLUE) } \\
\hline & Intercept $\mathrm{t}^{\mathrm{a}}$ & 2009 & 2010 & 2011 & 2012 \\
\hline Fruit weight (g) & $178.9 \pm 17.6^{\mathrm{b}}$ & 0 & $11.9 \pm 2.8$ & $50.3 \pm 2.8$ & $13.2 \pm 2.5$ \\
\hline Fruit skin color & $1.85 \pm 0.11$ & 0 & $0.06 \pm 0.02$ & $0.07 \pm 0.02$ & $-0.04 \pm 0.02$ \\
\hline Fruit surface texture & $2.54 \pm 0.14$ & 0 & $0.04 \pm 0.04$ & $-0.01 \pm 0.04$ & $-0.12 \pm 0.03$ \\
\hline Peelability & $2.23 \pm 0.16$ & 0 & $-0.12 \pm 0.04$ & $-0.20 \pm 0.04$ & $-0.30 \pm 0.03$ \\
\hline Flesh color & $2.48 \pm 0.08$ & 0 & $0.02 \pm 0.02$ & $0.01 \pm 0.02$ & $-0.07 \pm 0.01$ \\
\hline Pulp firmness & $2.72 \pm 0.15$ & 0 & $-0.05 \pm 0.04$ & $-0.15 \pm 0.04$ & $-0.14 \pm 0.03$ \\
\hline Segment firmness & $2.37 \pm 0.13$ & 0 & $-0.02 \pm 0.04$ & $-0.13 \pm 0.04$ & $-0.15 \pm 0.03$ \\
\hline Sugar content (Brix\%) & $11.4 \pm 0.4$ & 0 & $0.0 \pm 0.1$ & $-1.2 \pm 0.1$ & $-0.4 \pm 0.1$ \\
\hline Acid content $(\%)$ & $1.79 \pm 0.18$ & 0 & $0.03 \pm 0.02$ & $-0.03 \pm 0.02$ & $0.19 \pm 0.02$ \\
\hline
\end{tabular}

a The BLUE of each intercept includes the year effect of 2009

${ }^{b}$ The number following the plus-minus sign $( \pm)$ relates to the standard error of each estimate 
Table 5 Estimates of narrow-sense heritabilities (diagonal and in bold) and genetic (upper triangle) and phenotypic (lower triangle) correlations for the nine fruit-quality traits

\begin{tabular}{|c|c|c|c|c|c|c|c|c|c|}
\hline & Fruit weight & $\begin{array}{l}\text { Fruit skin } \\
\text { color }\end{array}$ & $\begin{array}{l}\text { Fruit surface } \\
\text { texture }\end{array}$ & Peelability & Flesh color & $\begin{array}{l}\text { Pulp } \\
\text { firmness }\end{array}$ & $\begin{array}{l}\text { Segment } \\
\text { firmness }\end{array}$ & $\begin{array}{l}\text { Sugar } \\
\text { content }\end{array}$ & $\begin{array}{l}\text { Acid } \\
\text { content }\end{array}$ \\
\hline Fruit weight & $0.77 \pm 0.01 \mathrm{a}$ & $-0.09 \pm 0.04$ & $-0.07 \pm 0.05$ & $-0.18 \pm 0.04$ & $-0.17 \pm 0.04$ & $-0.08 \pm 0.04$ & $0.18 \pm 0.05$ & $-0.19 \pm 0.03$ & $0.12 \pm 0.03$ \\
\hline Fruit skin color & $-0.06 \pm 0.03$ & $0.63 \pm 0.02$ & $-0.06 \pm 0.05$ & $0.12 \pm 0.05$ & $0.35 \pm 0.04$ & $0.03 \pm 0.05$ & $0.09 \pm 0.05$ & $0.08 \pm 0.04$ & $-0.08 \pm 0.03$ \\
\hline Fruit surface texture & $-0.09 \pm 0.03$ & $-0.03 \pm 0.03$ & $0.46 \pm 0.03$ & $0.06 \pm 0.06$ & $-0.03 \pm 0.06$ & $0.03 \pm 0.06$ & $0.28 \pm 0.06$ & $-0.04 \pm 0.05$ & $0.00 \pm 0.04$ \\
\hline Peelability & $-0.11 \pm 0.02$ & $0.04 \pm 0.03$ & $0.12 \pm 0.03$ & $0.54 \pm 0.02$ & $0.17 \pm 0.05$ & $0.38 \pm 0.05$ & $-0.04 \pm 0.06$ & $-0.07 \pm 0.04$ & $-0.13 \pm 0.04$ \\
\hline Flesh color & $-0.13 \pm 0.02$ & $0.28 \pm 0.02$ & $-0.01 \pm 0.03$ & $0.08 \pm 0.03$ & $0.63 \pm 0.02$ & $0.17 \pm 0.05$ & $0.12 \pm 0.06$ & $0.09 \pm 0.04$ & $-0.17 \pm 0.03$ \\
\hline Pulp firmness & $-0.09 \pm 0.03$ & $0.03 \pm 0.03$ & $0.07 \pm 0.03$ & $0.22 \pm 0.02$ & $0.17 \pm 0.03$ & $0.58 \pm 0.02$ & $0.02 \pm 0.06$ & $0.10 \pm 0.04$ & $0.01 \pm 0.04$ \\
\hline Segment firmness & $0.08 \pm 0.03$ & $0.05 \pm 0.03$ & $0.15 \pm 0.02$ & $0.04 \pm 0.03$ & $0.07 \pm 0.03$ & $0.05 \pm 0.03$ & $0.45 \pm 0.03$ & $0.30 \pm 0.05$ & $-0.07 \pm 0.04$ \\
\hline Sugar content & $-0.17 \pm 0.02$ & $0.08 \pm 0.02$ & $0.00 \pm 0.03$ & $-0.05 \pm 0.03$ & $0.08 \pm 0.03$ & $0.08 \pm 0.03$ & $0.16 \pm 0.02$ & $0.74 \pm 0.01$ & $0.02 \pm 0.03$ \\
\hline Acid content & $0.06 \pm 0.02$ & $-0.06 \pm 0.02$ & $0.02 \pm 0.02$ & $-0.10 \pm 0.02$ & $-0.12 \pm 0.02$ & $0.01 \pm 0.02$ & $-0.04 \pm 0.02$ & $0.03 \pm 0.02$ & $0.91 \pm 0.00$ \\
\hline
\end{tabular}

a The number following the plus-minus sign $( \pm)$ indicates the standard error of each estimate

Table 6 Illustration of best linear unbiased predictions (BLUPs) of breeding values for fruit weight in parental cultivars (upper) and F1 offspring (lower) evaluated in this study

\begin{tabular}{|c|c|c|c|c|c|}
\hline \multirow[t]{2}{*}{ Name } & \multirow[t]{2}{*}{ Number of relatives } & \multicolumn{4}{|c|}{ Fruit weight (g) } \\
\hline & & $G V^{a}$ & $\mathrm{BV}^{\mathrm{b}}$ & $\operatorname{Rank}^{\mathrm{c}}$ & Accuracy \\
\hline satsuma mandarin & 2057 & 155.6 & -23.3 & 639 & 0.95 \\
\hline sweet orange & 2015 & 161.2 & -17.7 & 550 & 0.94 \\
\hline ponkan & 1044 & 173.8 & -5.1 & 414 & 0.96 \\
\hline Kiyomi & 2061 & 197.9 & 19.0 & 221 & 0.95 \\
\hline Shiranuhi & 2063 & 201.6 & 22.7 & 195 & 0.96 \\
\hline 050317 & 2063 & 206.7 & 27.9 & 158 & 0.92 \\
\hline 050473 & 2105 & 120.0 & -58.9 & 1334 & 0.93 \\
\hline 050847 & 2063 & 165.7 & -13.1 & 490 & 0.89 \\
\hline 060084 & 2114 & 156.0 & -22.9 & 636 & 0.91 \\
\hline 060710 & 2095 & 199.0 & 20.1 & 213 & 0.92 \\
\hline
\end{tabular}

${ }^{\text {a }}$ Estimated genotypic values, calculated as the sum of the intercept and the breeding value

b Predicted breeding values

${ }^{c}$ Ranking for favorable fruit quality among all 2122 genotypes evaluated in this study based on predicted breeding value

d Accuracy, i.e., the reliability index of the predicted breeding values, was the inferred correlation between the true and predicted breeding values 